(C)2009 IEEE. Personal use of this material is permitted. However, permission to reprint/republish this material for advertising or promotional purposes or for creating new collective works for resale or redistribution to servers or lists, or to reuse any copyrighted component of this work in other works must be obtained from the IEEE. 


\section{On Supporting VoIP Traffic in Multi-hop IEEE 802.16 (d) Mesh Networks}

\author{
Lee-Chin Lau \\ Curtin University of Technology, \\ Miri, Malaysia.
}

\begin{abstract}
The IEEE 802.16(d) mesh mode is proposed for the next generation wireless mesh network. It specifies two mechanisms to schedule data transmission, namely centralized scheduling and distributed scheduling. In this paper, we evaluate the performance of VoIP traffic over an IEEE 802.16(d) mesh network using the distributed scheduling. The problems of the system in supporting voice traffic are identified. To solve these problems, we propose a Cycle-based Scheduling (CBS) as an extension to the original standard to improve the QoS of VoIP traffic whilst increase the channel utilization of the network. Our preliminary simulation results show that CBS can greatly improve the QoS support of the IEEE 802.16(d) mesh mode for VoIP traffic.
\end{abstract}

\section{INTRODUCTION}

Wireless Mesh Networks (WMNs) have attracted much research interests in recent years. In 2004, the IEEE 802.16 working group included the mesh mode in the standard [1]. The mesh mode defines two mechanisms to schedule data transmission in a collision-free manner. The centralized scheduling requires the mesh base station (MBS) to act as the central controller to determine the bandwidth allocation among all the mesh subscriber stations (MSSs). On the other hand, the distributed scheduling (DS) could be adopted based on the scheduling information of the two-hop neighbors. In this case, MSSs have to go through a three-way handshake $(\mathrm{TH})$ procedure to establish a connection between two nodes. In this paper, we will focus on DS as it exhibits better flexibility and efficiency on connection setup and data transmission.

With DS, MSSs schedule the transmission of control messages periodically and in a collision-free manner according to the mesh election algorithm specified by the IEEE 802.16(d) standard. For the detail operation of the distributed scheduler, interested reader may refer to [2]. Other related works have investigated the capacity estimation [3] and bandwidth allocation [4] of the system. A reservation protocol in serving VoIP traffic with QoS is proposed in [5]. However, these earlier studies neglected the problems of the system MAC-layer in handling voice traffic, particularly the delay incurred during the $\mathrm{TH}$ and the under-utilization of bandwidth. In this paper, we identify the above mentioned problems and propose a Cycle-based Scheduling (CBS) as an extension to the IEEE 802.16(d) Mesh MAC protocol to provide better QoS for VoIP traffic transmission.

\author{
Kah-Seng Chung, King-Sun Chan \\ Curtin University of Technology, \\ Perth, Western Australia.
}

The remainder of the paper is organized as follow. In section 2, we describe the TH of the IEEE 802.16(d) DS and identify the problems of the system in supporting VoIP flows. The proposed CBS is then described in section 3 with its performance evaluated in section 4. Finally, we draw the conclusion in section 5 .

\section{TH OF THE DISTRIBUTED SCHEDULING}

In an IEEE 802.16(d) DS network, nodes exchange Mesh Distributed Schedule (MSH-DSCH) control messages with neighboring nodes periodically. For bandwidth negotiation, the sender-receiver pairs have to perform a Request-GrantConfirm $\mathrm{TH}$ procedure through information elements (IEs) carried in the MSH-DSCH messages. The sender initiates the TH by transmitting a pair of request IE and availability IE. The former conveys the bandwidth request expressed as (slot range, frame range), where the slot range and frame range identify a contiguous range of mini-slots and frames, respectively. The availability IE, on the other hand, indicates a range of free mini-slots for the receiver to issue the grant.

Upon receiving the bandwidth request, the granting node replies a grant IE to notify its permission for the data transmission within the mini-slots specified by the received availability IE. Finally, before the actual data transmission can take place, the sender sends a confirm IE to complete the TH. During the procedure, the neighboring nodes of the senderreceiver pair are aware of the mini-slots status through the overheard IEs. As such, the TH can effectively avoid the data collision in the one-hop neighborhood of the sender-receiver pair.

The choice of the frame range specified in the request IE has a significant impact on the network performance. It has to be selected from a set of permitted value by the standard, e.g. $1,2,4,8,32$ and 128. It is intuitive that the larger the frame range value, the better the application level performance as a larger portion of continuous bandwidth is reserved for smooth data transmission.

However, for transmitting VoIP traffic, which generates a small amount of data periodically, reserving a large frame range will result in bandwidth wastage. In contrast, although a small frame range can increase the bandwidth utilization, it increases the voice packets delay as the $\mathrm{TH}$ has to be carried out more frequently. The packet delay is further increased drastically and unexpectedly with every failed or ineffective 


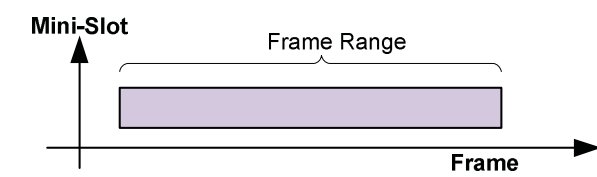

(a) Normal Bandwidth Reservation

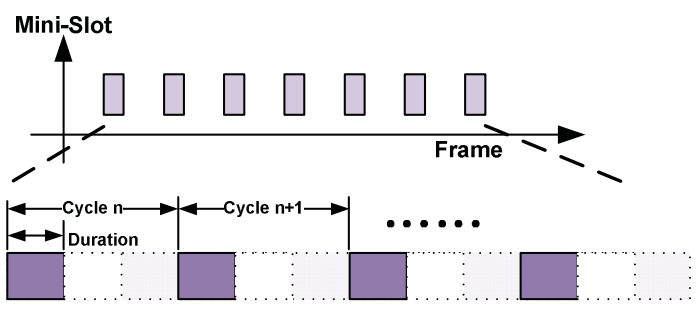

(b) Ideal CBS Reservation ( cycle $=3$, duration $=1$ )

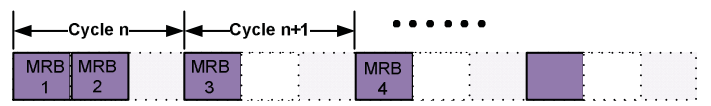

(c) Practical CBS Reservation ( $H E=0, M S H-D S C H-N U M=15)$

Fig 1: Bandwidth Reservation of (a) normal scheduling, (b) Ideal CBS, and (c) Practical CBS.

TH. Due to the distributive nature, the requesting node does not know the available resources at the granting node. Therefore, a $\mathrm{TH}$ fails if the specified mini-slots range in the Availability IE is unavailable at the granting node. As a result, the packets transmission has to be deferred until the next successful TH.

Furthermore, an ineffective TH can occur if the start frame of the Availability IE is set either earlier or too late than the $\mathrm{TH}$ completion time. The former causes the granted bandwidth before the completion of $\mathrm{TH}$ being wasted whereas the latter adds unnecessary packet delay before the transmission can start.

The above observations suggest that the IEEE 802.16(d) Mesh DS mode cannot service VoIP traffic efficiently. For example, selecting a large frame range results in bandwidth under-utilization whereas a small frame range increases the end-to-end delay of voice packets. Therefore, in this paper we propose a Cycle-based scheduling (CBS) to complement the MAC layer bandwidth reservation mechanism.

\section{PRoposed CYCLIC-BASED SCHEDULING}

The fundamental goal of the proposed CBS option is to request periodic link bandwidth, which is used particularly to serve constant bit rate (CBR) traffic with small amount of data such as VoIP. To implement CBS, all the IEs used in a TH are expanded with extra bits to specify two new parameters: cycle and duration. Cycle specifies the frame interval between two separate mini-slots reservations, whereas the duration specifies the consecutive number of frames that a mini-slot reservation lasts. The duration should be set less than or equal to the cycle at all time. When the duration and cycle are set to the same value, the CBS degenerates to normal bandwidth reservation.
Fig. 1 shows typical bandwidth reservation of a $\mathrm{TH}$, by setting the frame range value as 32. In a conventional 802.16(d) mesh network, the TH will result in a large block of reserved bandwidth as shown in Fig. 1(a). In contrast, Fig. 1(b) shows that CBS only reserves a small portion of bandwidth periodically according to the values of cycle and duration.

In CBS, a multiple pairs of request/availability IEs and a corresponding number of grant/confirm IEs are sent in every $\mathrm{TH}$. We call a group of mini-slots to be requested or being granted within a cycle as a mini-slot reservation block (MRB). Before performing a $\mathrm{TH}$, the requesting node has to calculate the number of MRBs (NMRB) according to:

$$
N M R B=\left\lfloor\frac{\text { frame_range }-1}{\text { cycle }}\right\rfloor+1+k .
$$

where $\mathrm{k}$ is a variable. In our implementation, the starting frame of the availability IE is set as early as possible to minimize the packet delay. Due to the ineffective $\mathrm{TH}$ elaborated in Section 2, the first MRB might expire upon the completion of a $\mathrm{TH}$. This prolongs the packet delay time because the packet transmissions are deferred until the next MRB takes place, which is (cycle - duration) frames later. To alleviate this problem, CBS requests an additional $k$ MRBs to transmit those packets that may be delayed due to the expiry of the first MRB. The variable $k$ is determine by the difference between the maximum and minimum number of frames required to complete a $\mathrm{TH}$, such that

$$
k=\max _{-} T H-\min \_T H .
$$

where

$$
\begin{aligned}
& \min _{-} T H=\left\lfloor\frac{2^{4+H E}}{M S H_{-} D S C H \_N U M}\right\rfloor \\
& \max _{-} T H=\left\lceil\frac{2^{4+H E}+\mathrm{NBR}_{2}-N U M}{M S H_{-} D S C H \_N U M}\right\rceil \text {. }
\end{aligned}
$$

The holdoff exponential value, $H E$ and $M S H \_D S C H \_N U M$ are both the network parameters that can be pre-configured. The $H E$ value decides the holdoff time of a node, defined as: $2^{H E+4}$, whereas $M S H \_D S C H \_N U M$ specifies the number of control slots used for MSH-DSCH messages transmission. $\mathrm{NBR}_{2} \mathrm{NUM}$ denotes the number of neighboring nodes in the two-hop neighborhood of the requesting node.

After determining the value of $N M R B$, the requesting node sends $N M R B$ pairs of request/availability IEs to the granting node. The starting frame (SF) for the $\mathrm{n}^{\text {th }}$ availability IE is calculated as follows:

$$
S F=\left\{\begin{array}{cc}
x+n+\min _{-} T H, & , 0 \leq n<k \\
x+\min _{-} T H+3(n-k) & , k \leq n \leq(N M R B-1)
\end{array}\right.
$$

where $x$ represents the frame that a TH is initiated.

Fig. 1(c) illustrates a practical bandwidth reservation of CBS in a chain network.

We further propose an extension, called CBS-ETH, which exploits an early TH (ETH) scheme. With this extension, a node can initiate the next $\mathrm{TH}$ before it uses up the granted MRBs. This can effectively hide the time overhead introduced by a $\mathrm{TH}$ from data packets and further reduce the packet delay time. We define the triggering timing of the ETH in terms of the number of remaining MRBs, as given by: 


$$
N R=\left\lceil\frac{\left(\max \_T H \times 2\right)+1}{\text { cycle }}\right\rceil .
$$

To guarantee a continuous MRB reservation, the NMRB and SF of CBS-ETH should be adjusted based on the followings:

$$
\begin{aligned}
& N M R B_{-} E T H=\left\lfloor\frac{\text { frame_range }-1}{\text { cycle }}\right\rfloor+1 \\
& S F_{\text {_ }} E T H=x^{\prime}+3+3 n, \quad 0 \leq n \leq N M R B \_E T H
\end{aligned}
$$

where $x$ ' represents the SF of the last MRB granted in the previous $\mathrm{TH}$.

It is to be noted that using CBS both the requesting node and granting node can optimally schedule their mini-slot reservations by exploiting more scattered separated mini-slots. This can effectively avoid bandwidth wastage in delivering VoIP traffic. Furthermore, a requesting node can obtain a number of mini-slot reservations to service the periodic voice data through single TH. As such, only the first transmitted voice packet of each $\mathrm{TH}$ will experience the time overheads introduced by $\mathrm{TH}$. The remaining voice packets arriving at the MAC layer can be transmitted quickly through the properly scheduled mini-slot reservations.

\section{PERFormance EVAluation}

\section{A. Simulation setup}

The performances of the original TH, CBS and CBS-ETH have been evaluated using the NCTUns network simulator [7] based on the parameter settings as shown in Table I.

Zero $\mathrm{HE}$ value is adopted to minimize the back-off time of nodes as suggested in [2]. In addition, the MSH_DSCH_NUM is set to the maximum value of 15 to minimize the $\mathrm{TH}$ completion time. Using the 64 QAM modulation scheme, each mini-slot can carry 323 bytes of data. The MAC-layer frame fragmentation function is disabled to reduce the implementation complexity. As such, each voice packet is sent as an individual and independent MAC-layer burst using one mini-slot. The values of cycle and duration are set to 3 and 1, respectively, as the most appropriate configuration to serve VoIP flows encoded with G.711 scheme at $30 \mathrm{~ms}$ packetization time.

The simulations make use of ten chain network topologies, each contains from 2 nodes up to 10 nodes. We assume that no channel error occurs during packet transmission.

\section{A. Performance metrics}

We define two performance metrics to evaluate network performances: 1) effective bandwidth utilization (EBU), and 2 ) the number of supportable hop counts (NSHC). The EBU shows the bandwidth utilization of a network defined as:

$$
E B U=\sum_{i=1}^{n} \frac{D B(i)}{R B(i)} \times 100 \%,
$$

where $D B(i)$ denotes the number of data bits transmitted by node $i$ and $R B(i)$ is the number of data bits reserved for node $i$.

On the other hand, the NSHC is determined by end-to-end delay and the packet loss of the VoIP flows. To ensure PSTNlike voice quality, the end-to-end delay and packet loss has to be kept below $150 \mathrm{~ms}$ and $3 \%$, respectively[8].
Table I: Simulation Parameters Settings

\begin{tabular}{|c|c|}
\hline Network Parameters & Value \\
\hline MSH-DSCH-NUM & 15 \\
\hline Number of Mini-slots/frame & 205 \\
\hline Hold-off Exponential Value (HE) & 0 \\
\hline Modulation/Coding Scheme & $64 \mathrm{QAM}-3 / 4$ \\
\hline Frame Duration & $10 \mathrm{~ms}$ \\
\hline VoIP Parameters & Value \\
\hline Codec & G.711u \\
\hline Data Rate & $64 \mathrm{Kbps}$ \\
\hline Packetization Time & $30 \mathrm{~ms}$ \\
\hline Packet Size & $280 \mathrm{Bytes}$ \\
\hline
\end{tabular}

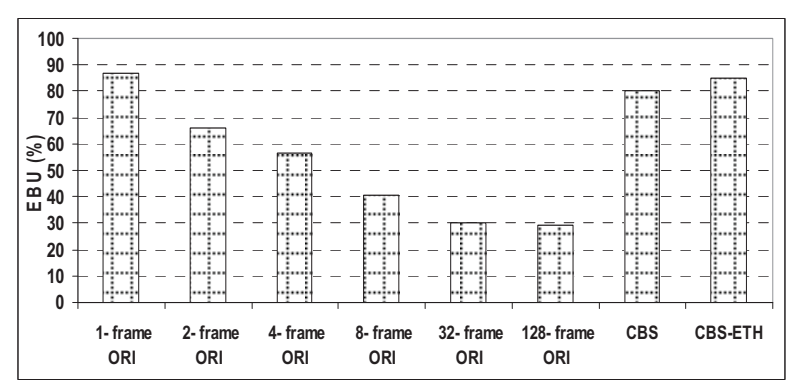

Fig. 2: EBU of Different Scheduling Schemes

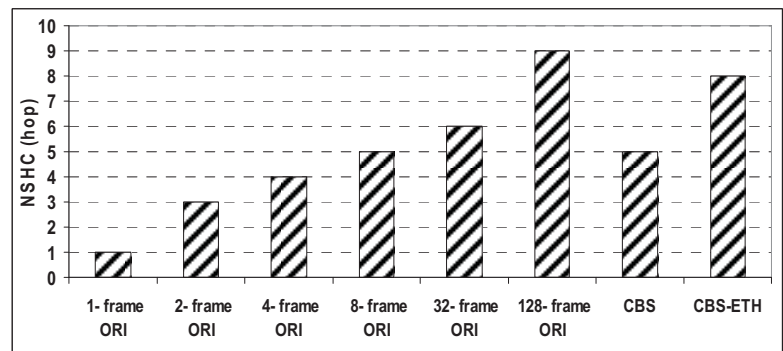

Fig. 3: NSHC of Different Scheduling Schemes

Therefore, in our simulation, a voice packet is regarded lost if it exceeds $150 \mathrm{~ms}$. We then define the NSHC as the maximum number of hops traversed by a VoIP flow between the source and destination nodes such that the resultant packet loss rate is less than $3 \%$.

\section{B. Performance with single voice flow}

In this section, a single VoIP flow is sent from the left-most node to the right-most node in each of the ten chain network topologies. For the original scheduling, we explore the impact of all the permitted frame range values on the system performance. But for the CBS and CSB-ETH, the frame range value is fixed as 32 .

Fig. 2 and Fig. 3 summarize the EBU and NSHC results of the three different scheduling schemes. Here, we denote the original 802.16 (d) TH using $n$ frame range as $n$-frame ORI. Also, the trends of the EBU results of all simulation cases are similar regardless of the size of the chain network. Therefore, we only present the average EBU results across all simulated cases.

It is observed that both the CBS-ETH and CBS achieve satisfactory EBU value, which is close to the $87 \%$ EBU achieved by 1 -frame ORI. Moreover, CBS-ETH and CBS significantly outperform 1-frame ORI on the NSHC value by a 


\section{On Supporting VoIP Traffic in Multi-hop IEEE 802.16 (d) Mesh Networks}

factor of 8 and 5, respectively. The low NSHC value of the 1frame ORI is due to the ineffective TH which increases the packet delay drastically. This problem, however, has been counteracted in the CBS by reserving additional MRB in every $\mathrm{TH}$ at the expense of a $7 \%$ drop in EBU. Nevertheless, the CBS-ETH can effectively prevent such ineffective TH through the ETH scheme and does not require extra MRB reservation. This explains why it achieves slightly better EBU than the CBS.

For the original scheduling scheme, it is shown that the EBU values decline drastically with the increasing of the frame range value. This reveals that though reserving a larger portion of bandwidth provides better QoS for VoIP flows, it causes more bandwidth wastage. For example, it is observed that the 128-frame ORI network can achieve the highest NSHC value of 9 hops, but its EBU value drops to $30 \%$. Overall, the results demonstrate the original $\mathrm{TH}$ cannot efficiently service the VoIP flows. There is tradeoff in choosing either a large or small frame range for the bandwidth reservation. However, none of the scheduling schemes in the original $\mathrm{TH}$ could provide satisfactory performance for voice type traffic.

On the other hand, the proposed CBS-ETH can support the VoIP flow up to an 8-hop network, while maintaining high EBU. In comparison, the CBS achieves a lower NSHC than the CBS-ETH. This is mainly due to the TH failure which could happen unexpectedly when the network size gradually increases, thus prolong the packet delay.

Overall, the proposed CBS schemes, particularly the CBSETH, outperform the original TH in supporting the VoIP flow. The CBS-ETH can successfully guarantee the QoS of VoIP flow while maintaining high link bandwidth utilization.

\section{Performance with multiple voice flows}

In this section, the performance of the CBS-ETH is evaluated under a multi-flow environment. For the simulation, we make use of different number of VoIP flows, each flow has a different starting time and call duration. The flow interarrival times follow the Poisson distribution with a mean value of $2.5 \mathrm{sec}$ while the call durations follow the exponential distribution with a mean value of $200 \mathrm{sec}$. The use of sufficiently long mean call duration ensures that most of the calls are active simultaneously.

At each node, voice packets are transmitted in a first-comefirst-serve manner. Each time before a node transmits its Request IEs, it has to determine the number of mini-slots to be requested based on the currently active VoIP flows. Fig. 4 shows that the EBU value of the CBS-ETH under multiple VoIP flows slightly decreases from $85 \%$ to an average of $81.6 \%$. This is attributed to varied arrival times of voice packets from different flows. Consider two VoIP flows A and $\mathrm{B}$, each arrives at frame $x$ and frame $x+8$, respectively.

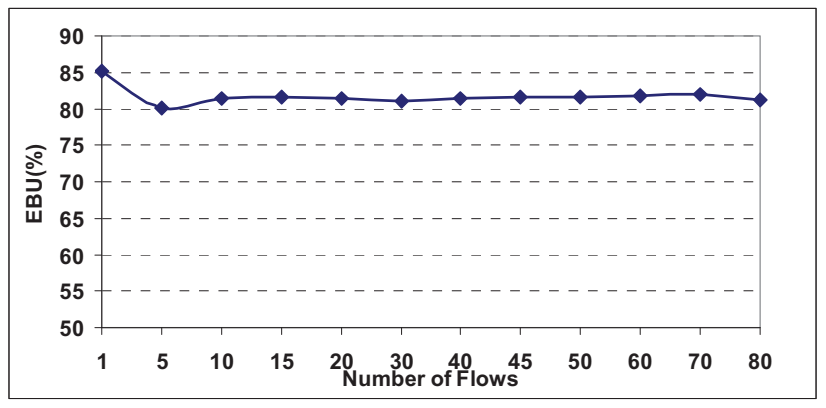

Fig. 4: EBU of CBS-ETH under Different VolP Load

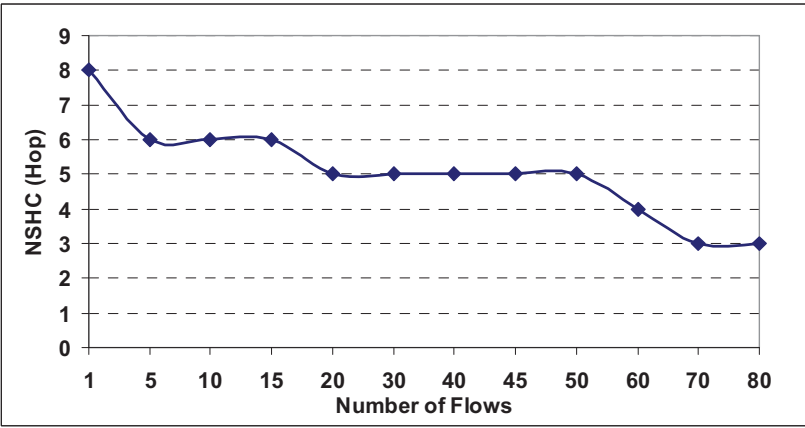

Fig. 5: NSHC of CBS-ETH under Different VoIP Load

As such, the requesting node has to initiate two separate THs to serve the two flows. According to equation (5), the last MRB of the first TH and the second TH will be scheduled at frame $x+31$ and frame $x+39$, respectively.

To avoid having to initiate separate $\mathrm{TH}$ for every individual voice flow, CBS-ETH adopts a request-merge strategy. It first scans the MAC-layer output queue to determine the number of active voice flows, denoted as Nms. Then it specifies Nms mini-slots in the request IEs that are going to be sent. Based on this request-merge scheme, after frame 31 , the node will request 2 mini-slots to serve flows $\mathrm{A}$ and $\mathrm{B}$ through a single $\mathrm{TH}$ (the third TH). However, the MRBs obtained in the third $\mathrm{TH}$ may partially overlap those of the second $\mathrm{TH}$, causing some of the MRBs to remain unused. This results in a small degradation in EBU as shown in Fig. 4. As this event only occurs once for every arrival of new VoIP flow and does not last more than 32 frames, the resultant bandwidth wastage is insignificant.

Fig. 5 shows the NSHC values obtained with CBS-ETH under different VoIP traffic load. It is observed that the NSHC value decreases slowly with an increase in VoIP flows. The reason is that the request-merge scheme requests MRBs based on the aggregate number of VoIP flows. As such, the scheduled MRBs may not be optimal for every of the VoIP flows which have different packet arrival times. This causes the performance degradation in terms of the end-to-end packet delay, thus the reduction in the NSHC value.

Furthermore, as the number of VoIP flows increases, this leads to an increase in the requested value of $\mathrm{Nms}$. Consequently, both the requesting node and granting node will encounter difficulty in obtaining the required MRBs. This causes more TH failures leading to a further increase in endto-end delay for th voice packets. One way of overcoming this shortcoming is to introduce an admission control 
Proceedings of the 15th Asia-Pacific Conference on Communications (APCC 2009)-191

mechanism to control the VoIP traffic load in the network. Such an extension, however, is out of the scope of this paper.

\section{CONCLUSION}

In this paper, the performance of the original IEEE 802.16(d) mesh protocol has been evaluated with regard to its capability in supporting VoIP traffic. It is shown that the protocol cannot achieve a satisfactory balance between network bandwidth utilization and end-to-end packet delay in delivering VoIP flows. Two enhancements, namely CBS and CBS-ETH, have been proposed to overcome the deficiency in the original IEEE 802.16(d) mesh protocol. Extensive simulation results show that both these schemes, particularly CBS-ETH, can provide QoS for the VoIP flows while maintaining satisfactory link bandwidth utilization.

\section{REFERENCES}

[1] "IEEE Standard for Local Metropolican Area Networks Part 16: Air Interface for Fixed Broadband Wireless Access Systems," IEEE Std 802.16-2004 (Revision of IEEE Std 802.16-2001).

[2] MinCao, M. Wenchao, Z.Qiang, and W.Xiaodong, "Analysis of IEEE 802.16 Mesh Mode Schedule Performance," IEEE Transaction on Wireless Communications, vol. 6, pp. 1455-1464, 2007.

[3] Y.Ge,C.K.Tham,P.Y.Kong, and Y.H.Ang, "Capacity Estimation for IEEE 802.16 Wireless Multi-hop Mesh Networks," in WCNC, 2008.

[4] C.Cicconetti, I.F.Akyildiz, and L.Lenzini, "Bandwidth Balancing in Multi-Channel IEEE 802.16 Wireless Mesh Networks," in IEEE Infocom, $26^{\text {th }}$ IEEE International Conference on Computer Communications, Anchorage, AK, 2007.

[5] C.Cicconetti, G.Vanessa, L.Lenzini, and E.Mingozzi, "End-to-End Bandwidth Reservation in IEEE 802.16Mesh Networks," in Mobile Adhoc and Sensor Systems, Pisa, 2006.

[6] C.Claudio, E.Alessandro, and L.Luciano, "Performance Evaluation of the Mesh Election Procedure of IEEE 802.16/WiMAX," in MSWiM'07,2007.

[7] S.Y.Wang, Y.C.Sung, S.M.Huang, "NCTUns Simulation Tool for WiMAX Modeling," in WICON Austin, Texas,USA, 2007.

[8] B.Yuval, "Fine-tuning Voice over Packet Services,", RADCOM Ltd.Ed. 